\title{
The Effect of Single Nucleotide Variations in the Transmembrane Domain of OATP IB I on in vitro Functionality
}

\author{
Wilma Kiander' • Kati-Sisko Vellonen² • Melina M. Malinen² • Mikko Gynther ${ }^{2,3}$ • Marja Hagström' •
} Madhushree Bhattacharya' • Seppo Auriola ${ }^{2}$ - Jan B. Koenderink ${ }^{4}$ - Heidi Kidron ${ }^{5}$

Received: 30 June 2021 / Accepted: 8 September 2021 / Published online: 13 October 2021

(c) The Author(s) 2021

\begin{abstract}
Purpose Organic Anion Transporting Polypeptide 1B1 (OATP1B1) mediates hepatic influx and clearance of many drugs, including statins. The SLCO1B1 gene is highly polymorphic and its function-impairing variants can predispose patients to adverse effects. The effects of rare genetic variants of SLCO1B1 are mainly unexplored. We examined the impact of eight naturally occurring rare variants and the well-known SLCO1B1 c.521C $>\mathrm{T}(\mathrm{V} 174 \mathrm{~A})$ variant on in vitro transport activity, cellular localization and abundance.
\end{abstract}

Methods Transport of rosuvastatin and 2,7-dichlorofluorescein (DCF) in OATP1B1 expressing HEK293

Supplementary Information The online version contains supplementary material available at https://doi.org/10. 1007/s11095-021-03107-8.

Heidi Kidron

heidi.kidron@helsinki.fi

1 Division of Pharmaceutical Biosciences, Faculty of Pharmacy, University of Helsinki, Viikinkaari 5E, 00014 Helsinki, Finland

2 School of Pharmacy, University of Eastern Finland, Kuopio, Finland

3 Pharmacy and Molecular Biotechnology, Ruprecht-Karls-University, Heidelberg, Germany

4 Department of Pharmacology and Toxicology, Radboud Institute for Molecular Life Sciences, Radboud University Medical Center, Nijmegen, the Netherlands

5 Division of Pharmaceutical Biosciences, Faculty of Pharmacy, University of Helsinki, Viikinkaari 5E, 00014 Helsinki, Finland cells was measured to assess changes in activity of the variants. Immunofluorescence and confocal microscopy determined the cellular localization of OATP1B1 and LC-MS/MS based quantitative targeted absolute proteomics analysis quantified the amount of OATP1B1 in crude membrane fractions.

Results All studied variants, with the exception of P336R, reduced protein abundance to varying degree. V174A reduced protein abundance the most, over $90 \%$ compared to wild type. Transport function was lost in G76E, V174A, L193R and R580Q variants. R181C decreased activity significantly, while T345M and L543W retained most of wild type OATP1B1 activity. P336R showed increased activity and H575L decreased the transport of DCF significantly, but not of rosuvastatin. Decreased activity was interrelated with lower absolute protein abundance in the studied variants.

Conclusions Transmembrane helices 2, 4 and 11 appear to be crucial for proper membrane localization and function of OATP1B1. Four of the studied variants were identified as loss-of-function variants and as such could make the individual harboring these variants susceptible to altered pharmacokinetics and adverse effects of substrate drugs.

\section{INTRODUCTION}

Organic Anion Transporting Polypeptide 1B1 (OATP1B1; encoded by SLCO1B1), a sinusoidal hepatic uptake transporter, plays a critical role in the pharmacokinetics of many widely used drugs such as cholesterol-lowering statins (1). EMA and FDA have issued guidelines on its study on pharmacokinetics during 
drug development, recognizing the importance of OATP1B1 in drug safety $(2,3)$.

Single nucleotide variations (SNV) in the SLCO1B1 gene can reduce plasma membrane expression or alter substrate affinity to the transporter, leading to altered pharmacokinetics of its substrate drugs. The well-characterized c.521 T $>$ C SNV reduces clearance and elevates plasma concentrations of many statins (4). Results of this can, in a dose-dependent manner, be muscle pain, weakness, and, in the worst-case, rhabdomyolysis. Compared to non-carriers, homozygous carriers of the c.521 $\mathrm{T}>\mathrm{C}$ variant are 30 times more susceptible to the development of simvastatin-induced myopathy (5).

The SLCO1B1 c.521 T $>\mathrm{C}$ variant is rather common: $32 \%$ of people with European ancestry are heterozygotes (TC genotype) and $4 \%$ are homozygotes (CC genotype) (6). This has enabled clinical association studies with good statistical power. With rare genetic variants, it is difficult to gather a patient group of sufficient size; nonetheless, their impact on drug safety can be as consequential (7). To overcome this issue, methods in in vitro-in vivo extrapolation (IVIVE) such physiologically based pharmacokinetic modelling (PBPK) incorporating in vitro data can help estimate the changes in in vivo pharmacokinetics and identify genotypes that could warrant caution when prescribing OATP1B1 substrate drugs (8).
The three-dimensional molecular structure of OATP1B1 has yet to be established and knowledge on the important substrate-binding sites is limited. OATP1B1 is predicted to form twelve transmembrane helices, with cytoplasmic N- and C-terminals. Sitedirected mutation studies have provided information on the effects of amino acid substitution on transporter function and identified transmembrane helices that are essential for transporter function (9). Based on computational predictions tools such as SIFT and PolyPhen, many of the naturally occurring genetics variants in the putative transmembrane region are deleterious $(10$, $11)$.

Here we characterized nine naturally occurring SNVs, where the amino acid substitution occurs in the putative transmembrane region of OATP1B1 (Fig. 1, Table I). The variants were chosen based on the changes in the characteristics of primary protein structure such as loss or gain of charge and location in critical transmembrane helices. We determined the cellular uptake of two OATP1B1 substrates, 2,7-dichlorofluorescein (DCF) and rosuvastatin, in OATP1B1 overexpressing HEK293 cells, and evaluated the cellular localization and quantified the protein expression of the variants. The SLCO1B1 c.521 T>C SNV served as a clinical reference variant. Our results provide new insight to amino acids critical to the transporter function and SNVs that
Fig. I Location of the selected single nucleotide variants in the predicted transmembrane helices of OATPIBI. The figure is based on the Uniprot entry Q9Y6L6 and generated with Protter (12)

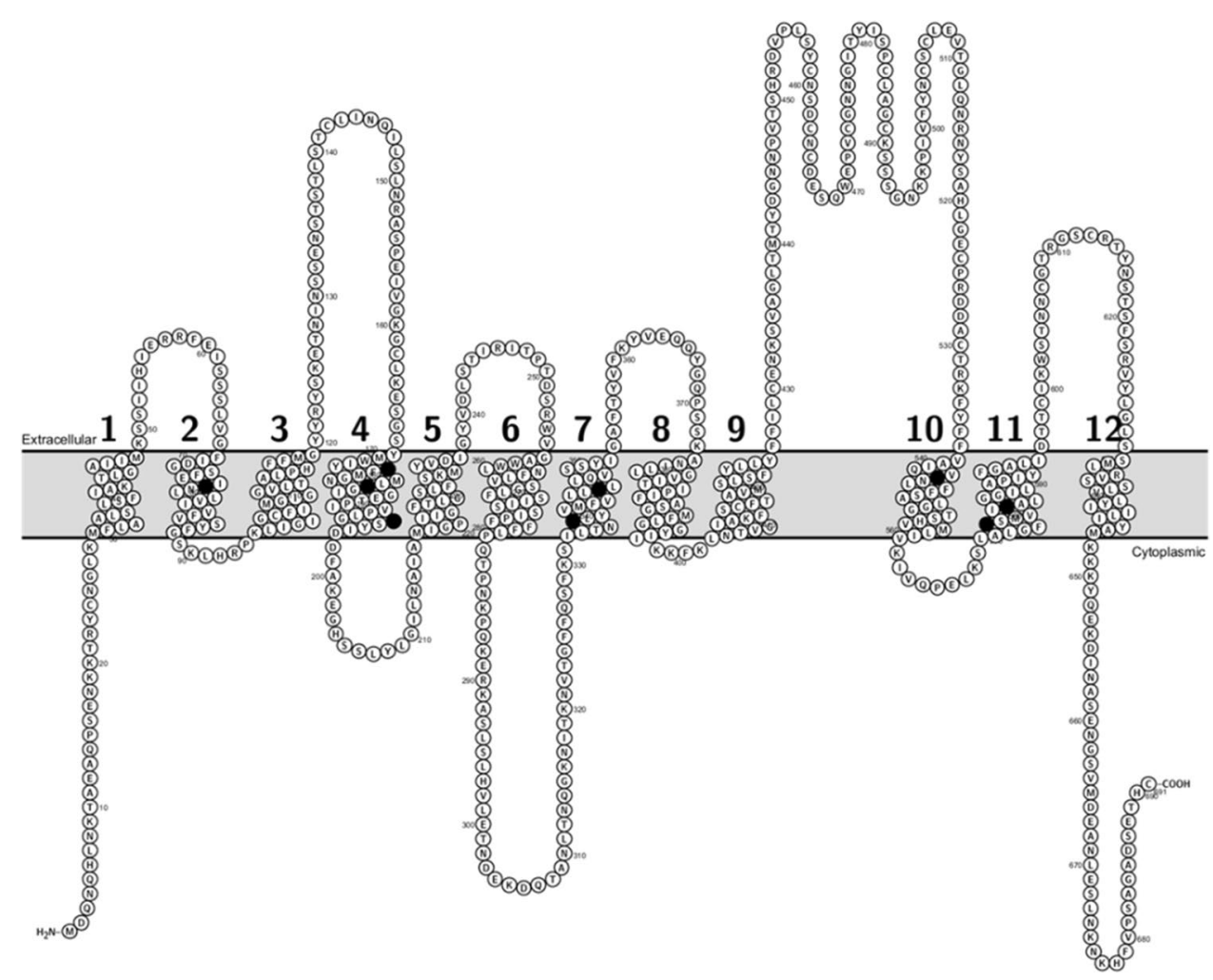


Table I The SLCOIBI single nucleotide variants included in the study, their prevalence and predicted consequences of the amino acid changes

\begin{tabular}{|c|c|c|c|c|c|}
\hline SNV & Amino acid change & Variant ID & Frequency $^{\mathrm{a}}$ & SIFT prediction ${ }^{b}$ & PolyPhen prediction ${ }^{c}$ \\
\hline c. $227 \mathrm{G}>\mathrm{A}$ & Gly76Glu & rs866376897 & ND & 0 (deleterious) & I (propably damaging) \\
\hline C.52I T>C & ValI74Ala & rs4|49056 & $0.133^{d}$ & 0 (deleterious) & 0.996 (propably damaging) \\
\hline$c .54 I C>T$ & Arg|8|Cys & rs|38965366 & $0.0000159^{e}$ & 0 (deleterious) & 0.99I (propably damaging) \\
\hline c.578 T>G & Leul93Arg & rs72559746 & ND & 0 (deleterious) & 0.863 (possibly damaging) \\
\hline c. $1007 C>G$ & Pro336Arg & rs72559747 & $0.000086 I^{f}$ & | (tolerated) & 0.044 (benign) \\
\hline c. $1034 C>T$ & Thr345Met & rs61760243 & $0.000385^{d}$ & 0.06 (tolerated & 0.523 (possibly damaging) \\
\hline c. $1628 \mathrm{~T}>\mathrm{G}$ & Leu543Trp & rs $7266 \mid 137$ & $0.00000796^{f}$ & 0 (deleterious) & 0.951 (propably damaging) \\
\hline c. $1724 \mathrm{~A}>\mathrm{T}$ & His575Leu & rs74700754 & ND & 0.01 (deleterious) & 0.142 (benign) \\
\hline c. $1739 \mathrm{G}>\mathrm{A}$ & Arg580Gln & rs763991908 & $0.0000333^{d}$ & 0 (deleterious) & 0.999 (propably damaging) \\
\hline
\end{tabular}

$\mathrm{ND}=$ not determined.

Frequency = Acquired from the gnomAD browser version 2.I.I (I3) https://gnomad.broadinstitute.org/

b SIFT Human Protein online service (I I) (http://sift.jcvi.org/www/SIFT_enst_submit.html).

c the Poly-Phen 2 service ( I0) (http://genetics.bwh.harvard.edu/pph2/) using the human SLCOIBI protein sequence (Uniprot entry Q9Y6L6) as the template.

d Global population.

e European (non-Finnish) and South Asian.

f East Asian.

may make their carriers susceptible to altered pharmacokinetics of OATP1B1 substrates.

\section{MATERIALS AND METHODS}

\section{Materials}

Fetal bovine serum (FBS), Hank's Balanced Salt Solution (HBSS), Dulbecco's Modified Eagle Medium (DMEM), Coomassie Plus protein assay reagent and HyClone SfX Insect cell medium were from ThermoFisher Scientific (Waltham, MA, USA). Q5® SiteDirected Mutagenesis Kit was from New England Biolabs (Ipswich, MA, USA). Oligomer (Helsinki, Finland) produced the mutagenesis primers. Toronto Research Chemicals (North York, ON, Canada) provided rosuvastatin hemicalcium and rosuvastatin-d6 sodium salt and DCF was from Santa Cruz Biotechnology (Dallas, TX, USA). Dithiothreitol and iodoacetamide were purchased from Sigma-Aldrich (Saint Louis, MO, USA). The peptide standards for MRM analysis were from JPT Peptide Technologies GmbH, Berlin, Germany. ProteaseMax surfactant, LyC endopeptidase and tosylphenylalanylchloromethyl ketone-treated trypsin were from Promega (Madison, WI, USA). All the other chemicals were purchased from Sigma-Aldrich (Saint Louis, MO, USA).

\section{Preparation of Plasmids Carrying SLCO IB I Variant Forms}

Q5® Site-Directed Mutagenesis Kit and PCR introduced the single nucleotide variants $(\mathrm{SNV})$ to the reference $S L C O 1 B 1$ gene (Genebank ${ }^{\mathrm{TM}}$ accession number AJ132573.1) in pENTR221 entry vector using the primers described in Supplementary table I. Sequencing service from GATC Biotech (Constance, Germany) verified the presence of the SNVs in the plasmids (data not shown). Baculoviruses carrying the wild type and mutated SLCO1B1 genes along with previously cloned gene for enhanced yellow fluorescent protein (eYFP, negative control) (14) were produced as described by Tikkanen et al. (15).

\section{Cell Culture and Protein Expression}

HEK293 cells were cultured in DMEM, high-glucose, GlutaMax culture medium supplemented with $10 \%$ FBS at $37{ }^{\circ} \mathrm{C}, 5 \% \mathrm{CO}_{2}$. Twenty-four hours prior to transduction with baculoviruses, $0.5 * 10^{6}$ cells were seeded in each well of 48-well plates (either poly-D-lysine coated ThermoFisher Scientific ${ }^{\mathrm{TM}} \mathrm{Nunc}^{\mathrm{TM}}$ or Corning ${ }^{\mathrm{TM}}$ CellBind $^{\mathrm{TM}}$ ). Sodium butyrate added with the viruses at a final concentration of $5 \mathrm{mM}$ (in-house optimization, data not shown) stimulated the expression of the proteins. 


\section{Cellular uptake assays}

The cellular uptake assay was conducted $48 \mathrm{~h}$ post-transduction on a heated $\left(37^{\circ} \mathrm{C}\right)$ orbital shaker plate. $500 \mu \mathrm{l}$ of transport buffer (HBSS with $4.17 \mathrm{mM} \mathrm{NaHCO} 3$ and 25 mM HEPES adjusted to $\mathrm{pH} 7.4$ with $\mathrm{NaOH}$ ) replaced the medium in the wells for a 3-min preincubation. After the removal of the buffer, uptake began with the addition of $125 \mu$ l of test solution containing test compound in transport buffer. Aspiration of the test solution stopped the uptake within the linear uptake phase for each substrate: 2 min for rosuvastatin and 15 min for 2,7-dichlorofluorescein (DCF). An immediate three-time wash with $500 \mu \mathrm{l}$ ice-cold transport buffer followed. The cells were lysed with $125 \mu \mathrm{l}$ of appropriate lysis buffer (3:1 methanol-water mixture containing rosuvastatin-D6 as an internal standard for rosuvastatin and $0.1 \mathrm{M} \mathrm{NaOH}$ in DCF samples). DCF was quantified by fluorescence measurement of the cell lysates (excitation $500 \mathrm{~nm}$, emission $528 \mathrm{~nm}$, bandwith $5 \mathrm{~nm}$ ) with multimode microplate reader Varioskan LUX (Thermo Fisher Scientific, Vantaa, Finland). The quantification of rosuvastatin is described in the following paragraph. The total protein was determined by mixing $10 \mu \mathrm{l}$ of cell lysate with $300 \mu \mathrm{l}$ Coomassie Plus reagent followed by absorbance analysis (595 nm) with Varioskan LUX.

Uptake of test substrates was normalized to total protein amount. Uptake into eYFP expressing cells (representing passive influx) was subtracted from uptake into OATP1B1 expressing cells yielding active OATP1B1 mediated transport. This transport activity in the OATP1B1 variant cells was then normalized to the wild type OATP1B1 cells.

\section{Rosuvastatin LC-MS/MS Analysis}

Liquid chromatography (LC) mass spectrometry (MS) measurements of rosuvastatin were carried out with Waters ultra-high pressure LC-MS/MS instrument (Waters, MA, USA). Rosuvastatin D6 served as an internal standard (ISTD). Liquid chromatography coupled with a Waters UPLC HSS T3 $(1.8 \mu \mathrm{m}, 2.1 \times 75 \mathrm{~mm})$ column at $40{ }^{\circ} \mathrm{C}(1.5 \mu \mathrm{l}$ injection volume $)$ separated the analytes. Mobile phase consisted of $0.1 \%$ of formic acid (Merck, Darmstadt, Germany) in ultrapure water (A) and $100 \%$ of LC-MS grade acetonitrile (Honeywell, Seelze, Germany) (B). Gradient elution started with $20 \%$ of B at $0-0.2 \mathrm{~min}$, continued with $20-95 \%$ B at $0.2-1.6 \mathrm{~min}$ and the complete run time was $5.5 \mathrm{~min}$ including column wash and equilibration with flow-rate as $0.3 \mathrm{~mL} / \mathrm{min}$.

The MS measurements were carried out using Waters Xevo TQ-S triple quadrupole mass spectrometer coupled with an electrospray ionization (ESI) on a positive mode. Optimized ms-parameters were: capillary $3.5 \mathrm{kV}$, cone voltage $84 \mathrm{~V}$ for rosuvastatin and $62 \mathrm{~V}$ for ISTD, source temperature $150{ }^{\circ} \mathrm{C}$ and desolvation temperature $500{ }^{\circ} \mathrm{C}$. Nitrogen was used as a desolvation gas $(900 \mathrm{~L} \mathrm{~h}-1)$ and a cone gas $(150 \mathrm{~L} \mathrm{~h}-1)$, argon as a collision gas. Multiple reaction monitoring quantified the analytes. The precursor and fragment ions for rosuvastatin were 482.19>258.12 (collision energy (CE) $32 \mathrm{~V}$ ) and 482.19>300.19 (CE $34 \mathrm{~V})$. For ISTD the ions were $488.17>264.17$ (CE $30 \mathrm{~V}$ ) and $488.17>306.24$ (CE $38 \mathrm{~V})$. The resulting data was analysed with Waters MassLynx V4.1 software.

\section{Immunofluorescence Staining and Confocal Microscopy}

The samples were prepared as described in Sjöstedt et al. (16) The used primary antibody (NB100-74,482, Novus Biologicals, Centennial, CO, USA) was used at a dilution of 1:500 in blocking solution and the secondary fluorescent antibody goat anti-mouse $\operatorname{IgG}(\mathrm{H}+\mathrm{L})$-Alexa Fluor 488 (ThermoFisher Scientific, USA) was used at a 1:200 dilution in blocking solution. 4',6-diamidino2-phenylindole (DAPI) at a concentration of $25 \mu \mathrm{g} / \mathrm{ml}$ visualized the nuclei. The variants were divided into to two batches and due to equipment failure, the microscope type needed to be switched between batches. The used microscopes were Leica TCS SP5 confocal microscope or Aurox Clarity laser free confocal (Aurox Ltd, Oxfordshire, UK) on Leica DM6000 microscope (Leica Microsystems, Wetzlar, Germany). The images were processed with Corel Paintshop Pro (version 23.1.0.27, Corel Corporation, Ottawa, ON, Canada). The immunofluorescence analysis was repeated on a new batch of HEK293 cells transduced with a separate batch of baculoviruses with one batch presented in these figures.

\section{Crude Membrane Extraction}

HEK293 cells were cultured in T175 flasks for $24 \mathrm{~h}$ prior to addition of sodium butyrate $(5 \mathrm{mM}$ final concentration) and baculoviruses containing either wild type or variant SLCO1B1. The cells were collected $48 \mathrm{~h}$ later and centrifuged $(3000 \mathrm{~g}, 15 \mathrm{~min})$. The cells were broken down with Dounce tissue homogenizer, resuspended in Tris-sucrose (TS) buffer (10 mM Tris-HEPES, $250 \mathrm{mM}$ sucrose, $\mathrm{pH}$ 7.4) and held on ice. A 30-min centrifugation $\left(3550 \mathrm{~g}, 4{ }^{\circ} \mathrm{C}\right)$ followed. The supernatant was separated and a further centrifugation step $(21,000 \mathrm{~g}$, $4{ }^{\circ} \mathrm{C}, 99 \mathrm{~min}$ ) resulted in a pellet containing the crude membrane. The protein sample was suspended in TS buffer and quantified as described previously. 


\section{LC-MS/MS Based Quantitative Targeted Absolute Proteomics (QTAP) Analysis}

The absolute protein expression of OATP1B1 in the crude membrane preparations was quantified using LC-MS/MS-based QTAP approach. The protein sample preparation and quantitation with targeted LC-MS was based on the method described by Uchida et al. (17). Briefly, $50 \mu \mathrm{g}$ of crude membrane preparations were solubilized, denatured with denaturing buffer, reduced with dithiothreitol and S-carbamoylmethylated with iodoacetamide. Then the alkylated proteins were precipitated with a methanol:chloroform:water mixture. Subsequent precipitates were then dissolved in $6 \mathrm{M}$ urea in $100 \mathrm{mM}$ Tris- $\mathrm{HCl}(\mathrm{pH} 8.5)$ and diluted fivefold with $100 \mathrm{mM}$ Tris-HCl (pH 8.5). 150 pmol of isotope-labeled peptide mixture (Supplemental table II) (SpikeTides, JPT Peptide Technologies GmbH, Berlin, Germany) serving as internal standard and ProteaseMax surfactant (final concentration $0.05 \%$ ) were added and the sample was then digested twice: the first with $1 / 100$ LysC endopeptidase and then with 1/100 TPCK-treated trypsin.

The resulting peptides in the samples were analyzed with 6495 QQQ MS (Agilent Technologies, Santa Clara, CA, USA) with 1290 HPLC system (Agilent Technologies) and AdvanceBio peptide Map Column, $2.7 \mu \mathrm{m}$, $2.1 \times 250 \mathrm{~mm}$ (Agilent Technologies) using HPLC method described earlier (18). MS conditions were: $30 \mu \mathrm{l}$ injection volume, ESI positive ion mode, the source temperature $210{ }^{\circ} \mathrm{C}$, drying gas (nitrogen) flow rate $15 \mathrm{~L} / \mathrm{min}$, nebulizer pressure 45 psi, the MS capillary voltage $3 \mathrm{kV}$ and dwell time $40 \mathrm{~ms}$.

The unique peptides for OATP1B1 and $\mathrm{Na}^{+} / \mathrm{K}^{+}-$ ATPase were selected according to the in silico peptide selection criteria published by Uchida et al., 2013 (17) and four to six MRM transitions were quantified for each isotope-labeled and unlabeled peptide (Supplementary Table II) (19). The SNVs did not alter the peptides in this sequence. Three transitions with the highest intensity were selected and the peak area ratios of the analyte peptides and their respective internal standards were compared using Skyline application (MacCoss Lab Software, Seattle, WA). The results of OATP1B1 expression are presented as relative to the $\mathrm{Na}^{+} / \mathrm{K}^{+}$-ATPase expression level and normalized to wild type.

\section{Data Analysis}

Cellular uptake assays were conducted in triplicates in two to four separate experiments. The average of these technical triplicates is considered one data point and the data are presented as their mean \pm SEM. The proteomics samples (presented as mean \pm SEM) were prepared from four and immunofluorescence analysis from two separate batches of HEK293 cells.

The homogeneity of variance in the results was established with Levene's test (IBM SPSS Statistics for Windows, Version 27.0. IBM Corp, Armonk, NY, USA). Based on these results, the statistical significance of the differences in activity and expression levels was determined with either one-way analysis of variance (ANOVA) with the Dunnett's post hoc test for multiple comparisons or Kruskal-Wallis one-way analysis of variance with Dunn's post hoc test for multiple comparisons (GraphPad Prism 6.05, GraphPad Software, San Diego, CA, USA). Maximum velocity $\left(\mathrm{V}_{\max }\right)$ and Michaelis-Menten constant $\left(\mathrm{K}_{\mathrm{m}}\right)$ values of rosuvastatin transport for selected variants were calculated with nonlinear regression from concentration-dependent assay data and extra-sum-of-squares F-test assessed their statistical significance. P-values below 0.05 were considered significant in all analyses.

\section{RESULTS}

A selection of nine naturally occurring SNVs of OATP1B1 were expressed in HEK293 cells and their effect on transport function was evaluated using two substrates. In addition, LC-MS/MS-based QTAP approach and immunofluorescence analysis was utilized to assess any changes in protein abundance or cellular localization of OATP1B1.

\section{2,7-dichlorofluorescein Cellular Uptake Assay}

The transport activity of the variants was first evaluated with $1 \mu \mathrm{M}$ DCF cellular uptake assay. DCF transport by G76E, V174A and L193R variants was nearly abolished, with activity less than $10 \%$ of wild type OATP1B1 (Fig. 2). Additionally, H575L and R580Q expressing cells showed significantly reduced activity: less than $25 \%$ of wild type.

The activity of R181C variant was also reduced significantly, but it was still roughly half of the wild type. T345M and L543W variants showed no significant alteration, while in P336R expressing cells transport was on average $172 \%$ of the wild type OATP1B1 transport.

\section{Rosuvastatin Cellular Uptake Assay}

For evaluation with a clinically relevant OATP1B1 substrate, rosuvastatin, the variants were divided into two groups. The six variants exhibiting reduced cellular uptake of DCF were tested for rosuvastatin transport 


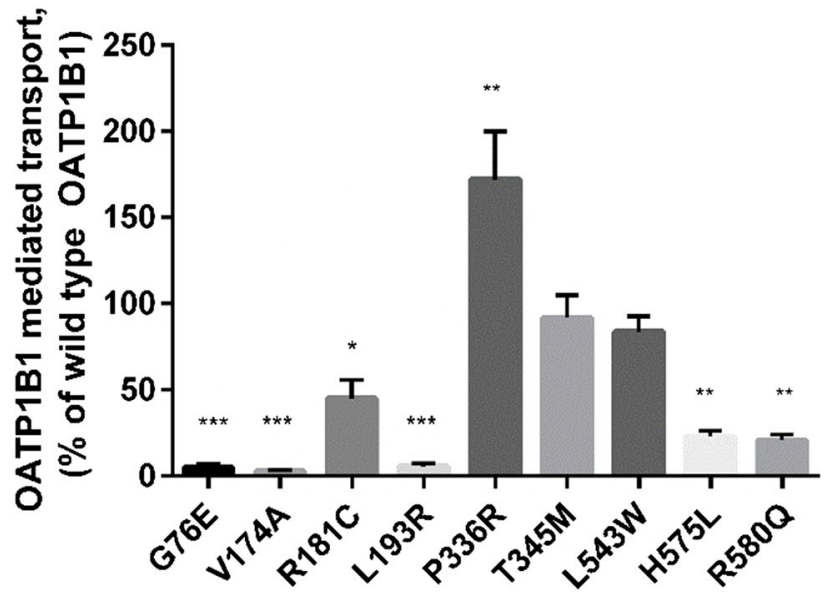

Fig. 2 OATPIBI mediated transport of I $\mu M$ DCF into variant OATPIBI expressing HEK293 cells over 15 min. Results are calculated as averages of four independent experiments conducted in triplicates and represented as $\%$ of wild type OATPIBI transport \pm SEM $(n=4)$. Statistical difference to the wild type is indicated as: $* * * *=P \leq 0.001$, ** $=\mathrm{P} \leq 0.01$, * $=\mathrm{P} \leq 0.05$

with three concentrations $(5,30,50 \mu \mathrm{M})$ (Fig. 3) and the rest were subjected to full concentration dependency assay (0.5-70 $\mu \mathrm{M})$ (Fig. 4).

Much like with DCF transport, G76E, V174A, L193R and $\mathrm{R} 580 \mathrm{Q}$ variants caused over $90 \%$ reduction in transport of rosuvastatin compared to the wild type (Fig. 3). No active transport was observed in V174A and R580Q expressing cells tested with 5 and $50 \mu \mathrm{M}$ rosuvastatin. The R181C variant performed similarly in transport of both test substrates, being significantly lower than in the wild type expressing cells. Curiously, the $\mathrm{H} 575 \mathrm{~L}$ variant transported rosuvastatin better than DCF. Increase in

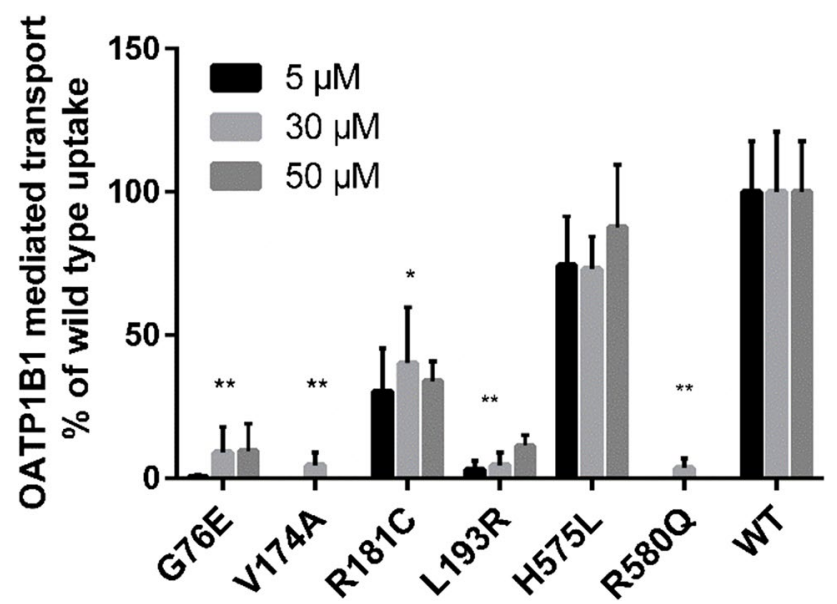

Fig. 3 OATPIBI mediated transport of rosuvastatin into variant OATPIBI expressing HEK293 cells in 2 min. Results are calculated as averages of two to four experiments conducted in triplicates and represented as \% of wild type OATPIBI transport \pm SEM $(n=2-4)$. Statistical difference to the wild type is indicated as: $* *=P \leq 0.01$, $*=P \leq 0.05$

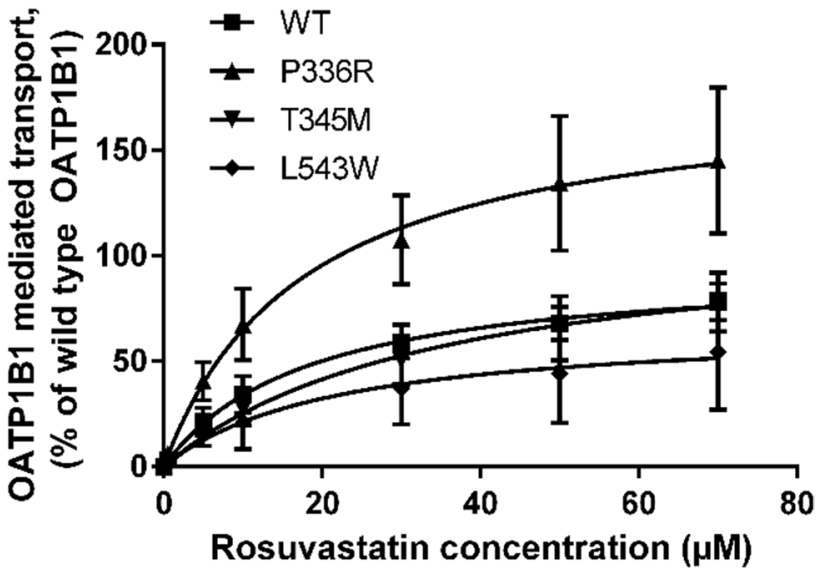

Fig. 4 Kinetics of OATPIBI mediated rosuvastatin transport into variant OATPIBI expressing HEK293 cells for 2 min. Results are calculated as averages of three experiments conducted in triplicates and represented \% of wild type (WT) OATPIBI transport \pm SEM $(n=3)$

rosuvastatin concentration resulted in no clear change in any of the studied variants.

The P336R variant transported rosuvastatin at a significantly higher velocity with $\mathrm{V}_{\max } 180 \%$ of the wild type, $\mathrm{P}<0.01$ (Fig. 4). T345M substitution led to no significant change in transport velocity and while $\mathrm{L} 543 \mathrm{~W}$ reduced transport, resulting in a $\mathrm{V}_{\max }$ value of $67 \%$ compared to the wild type, this was not statistically significant. There was no significant change observed in the $\mathrm{K}_{\mathrm{m}}$ values of the variants compared to the wild type (Table II).

\section{Quantitative Targeted Absolute Proteomics Analysis}

Crude membrane protein preparations of the wild type or variant OATP1B1 expressing HEK293 cells were analyzed using LC-MS/MS based quantitative targeted absolute proteomics (QTAP) approach to quantify OATP1B1. After normalization to $\mathrm{Na}^{+} / \mathrm{K}^{+}$-ATPase, the abundance of OATP1B1 in the variant samples were compared to the wild type (Fig. 5). The absolute amounts of OATP1B1 in the samples is listed in Supplementary table III.

With the exception of P336R, all of the studied SNVs resulted in reduced expression of OATP1B1 in HEK293 cells (Fig. 5). G76E, V174A, L193R and R580Q variant samples had the lowest (10-40\% of wild type, statistically significant) amount of OATP1B1 protein. The protein levels were compared to the transport activity of the variants and plotted in Fig. 6 . 
Table II Compilation of results. Uptake and abundance data are normalized to wild type (WT). Maximum velocity of transport $\left(\mathrm{V}_{\text {max }}\right)$ of the tested variants is normalized to the calculated $\mathrm{V}_{\max }$ of WT OATPIBI

\begin{tabular}{|c|c|c|c|c|c|}
\hline Variant & $\begin{array}{l}\text { I } \mu M \text { DCF uptake } \\
(\% \mathrm{WT}) \pm \text { SEM }\end{array}$ & $\begin{array}{l}5 \mu \mathrm{M} \text { Rosuvastatin } \\
\text { uptake }(\% \mathrm{WT}) \pm \mathrm{SEM}\end{array}$ & $\begin{array}{l}\text { Rosuvastatin } \mathrm{K}_{\mathrm{m}}{ }^{\mathrm{a}}(\mu \mathrm{M}) \\
(95 \% \mathrm{Cl})^{\mathrm{b}}\end{array}$ & $\begin{array}{l}\text { Rosuvastatin } \bigvee_{\max }{ }^{c} \\
(\% \mathrm{WT})(95 \% \mathrm{Cl})\end{array}$ & $\begin{array}{l}\text { OATPIBI abundance } \\
(\% \mathrm{WT}) \pm \text { SEM }\end{array}$ \\
\hline WT & 100 & 100 & | 8.7 (9.9 to 27.6$)$ & 100 & 100 \\
\hline G76E & $4.7 \pm 2.5$ & $0.5 \pm 0.5$ & ND & ND & $24.8 \pm 5.5$ \\
\hline VI74A & $2.6 \pm 0.8$ & $0 \pm 0$ & ND & ND & $9.7 \pm 1.7$ \\
\hline $\mathrm{RI} / 8 \mathrm{C}$ & $45.2 \pm 10.5$ & $30.3 \pm 15$ & ND & ND & $71.1 \pm 24.9$ \\
\hline LI93R & $5.46 \pm 1.9$ & $3 \pm 3$ & ND & ND & $29.8 \pm 4.6$ \\
\hline P336R & $172 \pm 28$ & $216.7 \pm 47.8$ & I 7.7 (0.0 to 37.9) & 179.9 ( 1 | 0.3 to 249.5$)$ & $101.4 \pm 35.9$ \\
\hline T345M & $92.1 \pm 12.8$ & $71.7 \pm 6.8$ & $35.2(0.0$ to 78.8$)$ & | 4.3 (5 |.| to |77.5) & $65.1 \pm 22.3$ \\
\hline L543W & $83.9 \pm 8.9$ & $51.5 \pm 29.9$ & $21.6(0.0$ to 78.0$)$ & $67.3(3.1$ to 131.4$)$ & $47.2 \pm 9.9$ \\
\hline H575L & $22.8 \pm 3.4$ & $74.3 \pm 17.2$ & ND & ND & $58.7 \pm 20.6$ \\
\hline R580Q & $20.8 \pm 3.3$ & $0 \pm 0$ & ND & ND & $36 \pm 3.5$ \\
\hline
\end{tabular}

$\mathrm{ND}=$ not determined

$\mathrm{a}=$ Michaelis-Menten coefficient.

$b=95 \%$ confidence interval.

$\mathrm{c}=$ maximum uptake velocity.

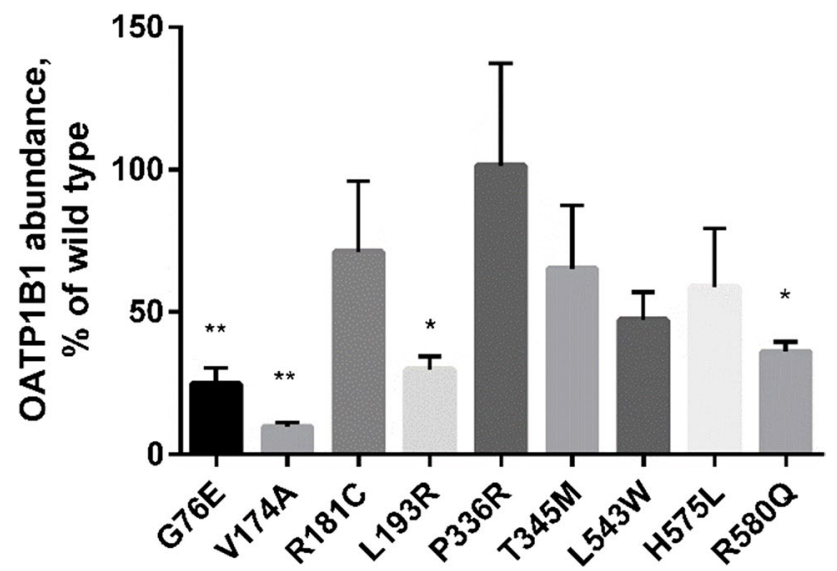

Fig. 5 LC-MS/MS proteomics analysis of 50 g HEK293 crude membrane preparations expressing wild type or variant OATPIBI. Abundance of OATPIBI was quantified in 4 independent samples, normalized to $\mathrm{Na}^{+} / \mathrm{K}^{+}$-ATPase and compared to wild type (I00\%) and presented as average \pm SEM $(n=4)$. *** $=P \leq 0.01, *=P \leq 0.05$

\section{Immunofluorescence staining and confocal microscopy}

Immunofluorescence and microscopy provided additional qualitative information on the localization of the OATP1B1 protein in control (non-transduced or eYFP expressing HEK293), wild type (WT) and variant OATP1B1 expressing HEK293 cells. Wild type OATP1B1 virus transduced cells showed typical concentrated band-like green color (Figs. 7 and 8). No green label was present in non-transduced HEK293 cells. The green color in the eYFP expressing cell sample is likely to be derived from the expressed fluorescent protein.

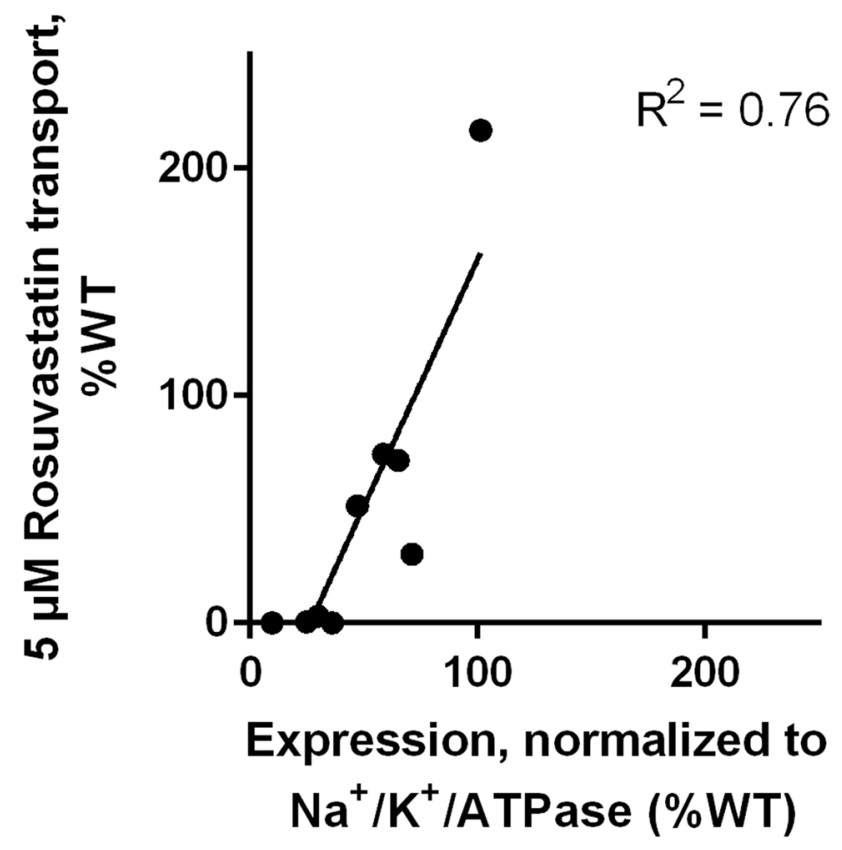

Fig. 6 Abundance of OATPIBI in crude membrane preparations compared to the observed OATPIBI mediated rosuvastatin transport. The line shows linear regression

In the G76E expressing cells, OATP1B1 was abundant yet it was not concentrated in similar band-like manner as in the wild type cells. In V174A, H575L, L193R and L543W variant cells the expression of OATP1B1 protein is appears lower than in the wild type (Fig. 7 and 8). Instead, in the R181C, R580Q, P336R 

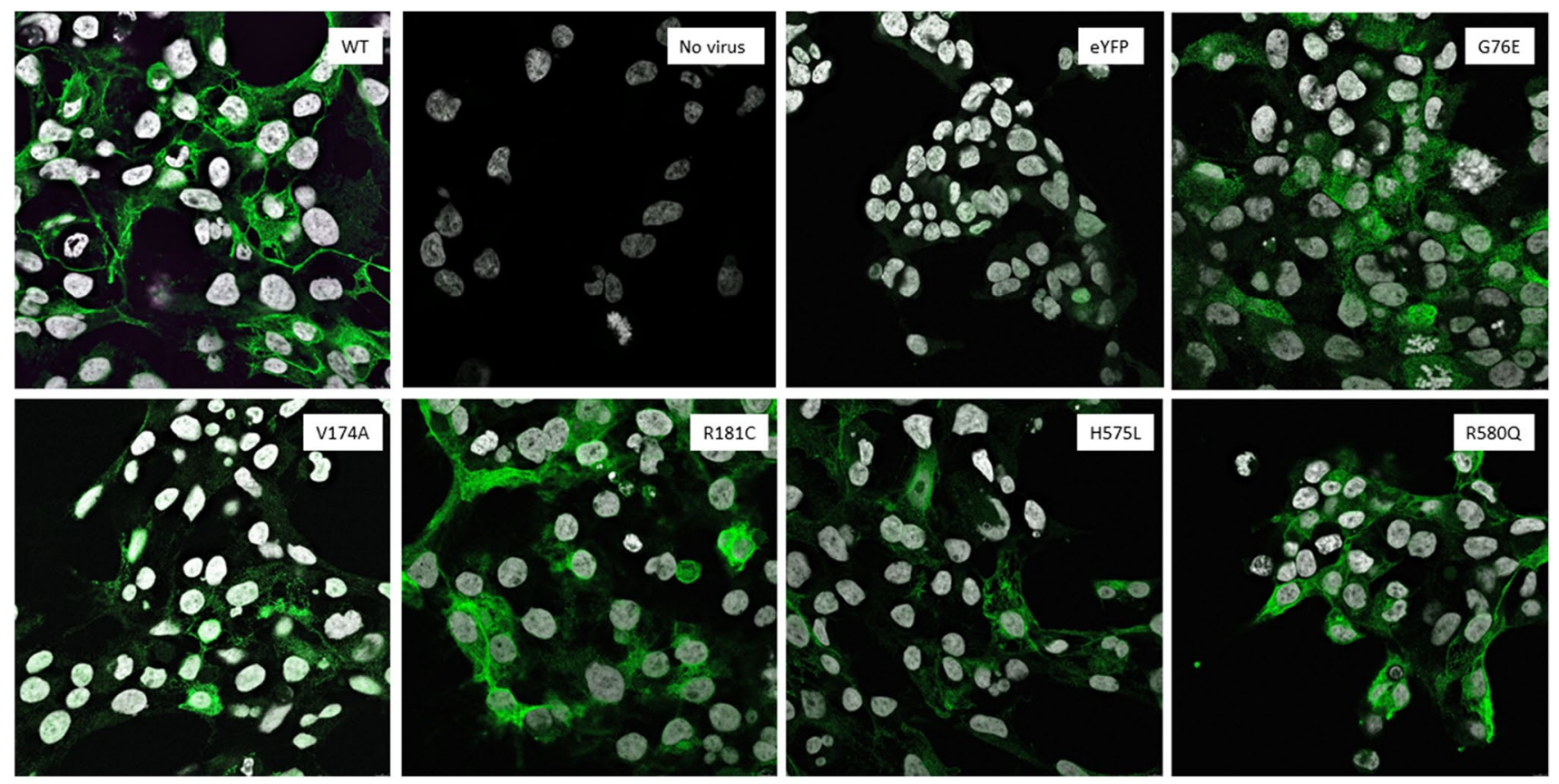

Fig. 7 Localization and expression of OATPIBI protein in representative baculovirus transduced HEK293 cells. OATPIBI was labeled with mouse NB 100-74,482 primary antibody and detected with goat anti-mouse AlexaFluor488 secondary antibody (green) using Leica TCS SP5 confocal microscope. The nuclei are visualized with DAPI (grey). Non-transfected (no virus) and eYFP expressing HEK293 cells were used as negative controls
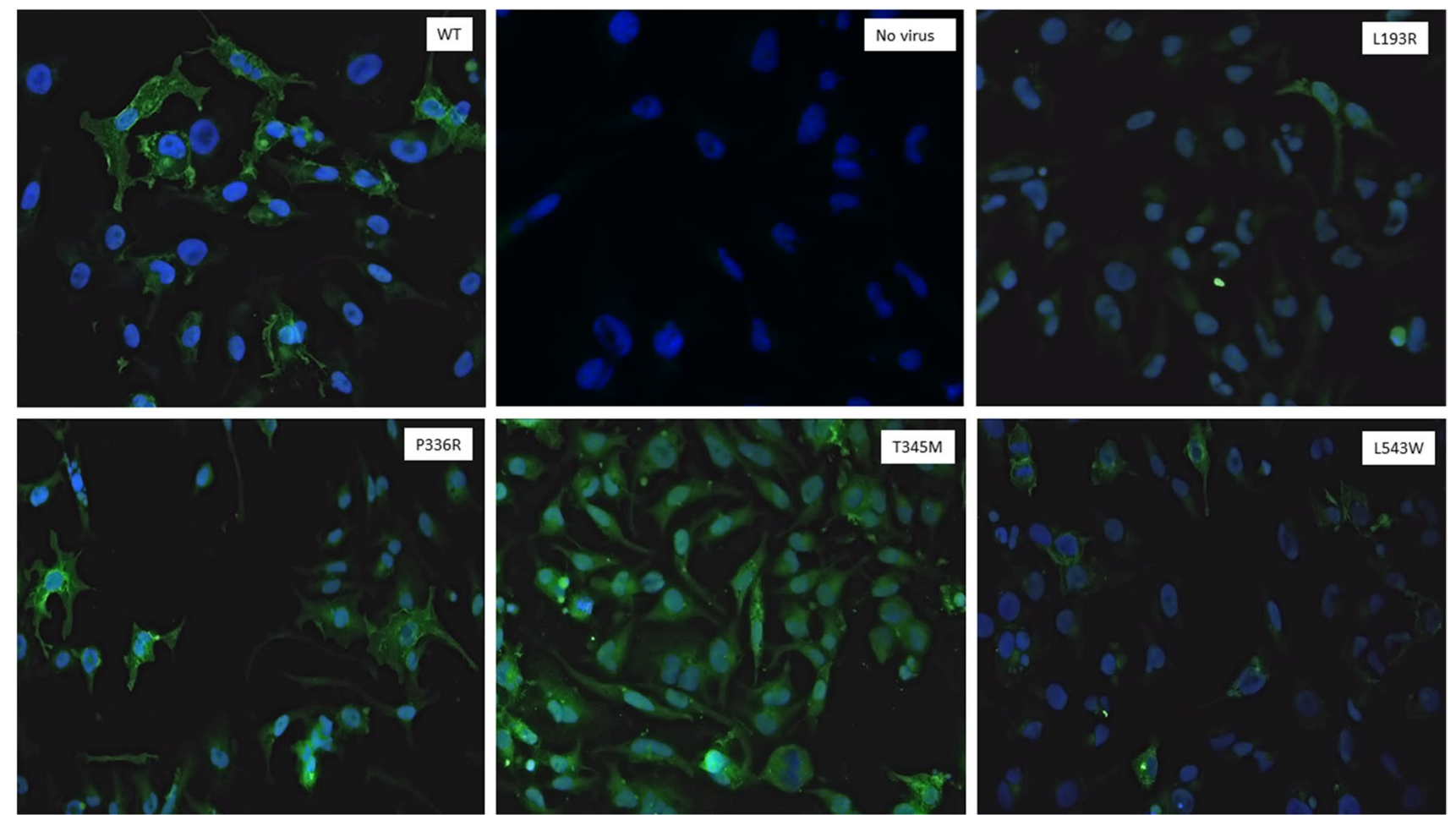

Fig. 8 Localization and expression of OATPIBI protein in representative baculovirus transduced HEK293 cells. OATPIBI was labeled with mouse NBI 00-74,482 primary antibody and detected with goat anti-mouse AlexaFluor488 secondary antibody (green) using Aurox Clarity laser free confocal on Leica DM6000 microscope. The nuclei are visualized with DAPI (in blue). Non-transfected HEK293 cells were used as negative control 
and $\mathrm{T} 345 \mathrm{M}$ variants the abundance and localization of OATP1B1 appear comparable to the wild type (Fig. 7 and 8 ).

\section{Discussion}

In this study, OATP1B1 transport activity and localization of nine naturally occurring SNVs were characterized in vitro. G76E, R181C, T345M, H575L and R580Q were, to our knowledge, characterized for the first time. A trend (linear regression $\mathrm{R}^{2} 0.76$ ) was observed between protein expression levels and activity for the studied variants: all loss of function variants had levels below $50 \%$ of wild type OATP1B1 (Fig. 6). This does not, however, fully explain the transport activities: for example P336R enhanced transport activity, despite similar abundance of OATP1B1.

In order to compensate for the variability in the used expression system, we used at least two independent batches of baculoviruses in the experiments and performed several independent experiments. Virus titer or multiplicity of infection (MOI) was not determined. Instead, the comparability of the variants was controlled by producing them at the same time with the wild type baculoviruses with standardized amount of DNA in each batch and the variants were only compared to the batch specific wild type virus. The amount of virus was tittered in in-house optimization experiments to achieve maximum transduction and the same ratio was used in all the experiments. The same protocol has previously been used by Sjöstedt et al. (16). We performed several experiments with different virus batches and given the low variability level of the results (Fig. 2 and 5), we are confident that the observed changes in the transporter function and expression are due to these SNVs and not variation in the transduction. Phenol red in known to inhibit OATP1B1 (20). While it is included in the cell culture medium, the medium in removed and its traces washed away with phenol red free transport buffer prior to initiation of uptake and consequently should not interfere with the assay.

The transmembrane helix 2, where G76E is located in, was previously shown to be important for transporter localization and function. SLCO1B1 c.211G > A, a variant that changes glycine to arginine at position 71 , reduces OATP1B1 mediated methotrexate transport severely in vitro and is associated with reduced methotrexate clearance (7). Substitutions of amino acids to alanine at positions 70, 73, 74 and 76 reduced in vitro transport of estrone-3- sulfate (E3S) significantly (21). Similarly, in our study the transport of both DCF and rosuvastatin into G76E variant cells was abolished and immunofluorescence staining showed altered localization compared to the wild type. Moreover, a nearly $75 \%$ reduction in OATP1B1 protein abundance was observed. The negative charge of glutamate substitution is more drastic than the previously mentioned alanine substitution, and it is reasonable to assume that both impair folding of the protein into its correct secondary structure. Taken altogether, the data provide evidence that the glycine in position 76 is vital.

Three of the studied variants are located to the putative transmembrane helix 4 . The SLCO1B1 c.521 T $>\mathrm{C}$ (V174A) genotype has resulted in increase in plasma AUC levels of e.g. rosuvastatin $(22,23)$. The in vitro data on this variant, nonetheless, is more conflicting. Semi-quantitative Western blot analysis of liver crude membrane fractions of heterozygote c.521 T $>C$ patients showed no difference in OATP1B1 abundance compared to reference (24). On the other hand, liver samples of the same genotype in another study analyzed with LC-MS/MS showed moderate decrease in OATP1B1 abundance compared to reference (8). It should be noted though, that the heterozygote genotype is not comparable to over-expressing systems, which correspond to the homozygote genotype. Nevertheless, the data from over-expressing systems is also contradictory. The cell-surface expression level of V174A was decreased in Western blot of biotinylated HeLa cell samples (25) and in immunocytochemical analysis of HEK293 and HeLa cells (26), while no change was observed in Western blot analysis of HEK293 and HeLa (both whole cell and biotinylated) samples in other studies (27-29). Differences in these results can arise from used cellular expression system, sample preparation and analysis methods. To our knowledge, this study is the first to quantify OATP1B1 V174A in an over-expressing system with a LC-MS/ MS proteomics method. We observed $>90 \%$ decrease in protein amount in the crude membrane fractions of OATP1B1 V174A compared to the wild type, which is in accordance to a meta-analysis, where the relative abundance of V174A OATP1B1 was $37 \%$ of the wild type OATP1B1 (30).

The in vitro transport activity of $\mathrm{V} 174 \mathrm{~A}$ has also varied between studies. The transport of E3S and estradiol-17 $\beta$ glucuronide $(\mathrm{E} 217 \mathrm{G})$ was not altered in two studies (27, 28 ) while it was reduced in others, along with rosuvastatin transport $(25,31)$. For the most part, the results have been consistent with the expression levels in those studies. Crowe et al., however, reported significantly lower accumulation of E217G in V174A expressing HEK293 cells but no change in cell surface expression (29). In our study, the activity was abolished with both tested substrates and interrelated with the protein expression levels. 
The arginine in position 181, also located in transmembrane helix 4, is conserved throughout the OATP1 family (32). In a previous study, lysine substitution at this position resulted in $90 \%$ reduction of OATP1B1 expression, while the reduction with alanine and histidine substitution was only 20 and $40 \%$, respectively (33). In our study, the protein expression of R181C was reduced by $30 \%$ compared to the wild type and transport of both tested substrates was less than $50 \%$ of wild type. Localization in immunofluorescence samples, instead, appeared comparable to wild type. These results suggest that the arginine is more important for the transport function of OATP1B1 than for stable protein expression.

L193R, the third of the studied variants in transmembrane helix 4, reduced the transport of both tested substrates severely, and the amount of variant protein was only $40 \%$ of the wild type. Immunofluorescence results also suggest lower abundance and plasma membrane localization compared to the wild type. This is in line with previous characterization of L193R, where the transport of sulfobromophthalein, E217G and cholyltaurine was abolished and Western blot analysis showed markedly reduced localization in crude membrane samples of both transfected MDCKII cells and a human liver sample (34).

The SNVs exhibiting increased or unaltered activity (P336R and T345M) are both found in the putative transmembrane helix 7 (Fig. 1). T345M did not alter either the activity or the localization or abundance of OATP1B1. Proline at the position 336 is a part of the $\mathrm{NPxY}$ motif involved in sorting of basolateral membrane proteins (35). This motif is conserved in OATP1B1, OATP1B3, OATP1C1 and OATP1A2. Alanine substitution decreased transport activity moderately (35). Clinical data on P336R is sparse: a single subject with the P336R variant had a high pravastatin AUC value and low total and non-renal clearance compared to the reference genotype (36). In vitro P336R substitution did not change or only slightly enhanced the transport of E3S, pravastatin, atorvastatin, cerivastatin and simvastatin (26). In our study, however, the activity of the P336R variant was significantly increased: DCF transport was $172 \%$ and the $\mathrm{V}_{\max }$ of rosuvastatin transport was $180 \%$ of wild type. The protein amount, however, did not differ from the wild type and the localization of the variant appeared comparable to wild type. The increase in $\mathrm{V}_{\max }$ without an increase in $\mathrm{K}_{\mathrm{m}}$ or expression levels suggests that this variant has a higher catalytic turnover rate than the wild type OATP1B1.

L543W is the only variant in our study that is located to transmembrane helix 10 (Fig. 1). It was first discovered in a Japanese man suffering from rhabdomyolysis
(37). In our study, the uptake of DCF was not altered and rosuvastatin $\mathrm{V}_{\max }$ was reduced moderately. This in accordance with the first in vitro characterization, where this variant did not alter the uptake of E3S but reduced pravastatin $\mathrm{V}_{\max }$ (no change in $\mathrm{K}_{\mathrm{m}}$ ) (38). Cell-surface biotinylation and Western blot analysis showed normal expression and localization on the plasma membrane in HEK293 cells. Our QTAP analysis, conversely, revealed reduced $(47 \%)$ protein expression compared to the wild type. This was also apparent in the immunofluorescence samples.

H575L and R580Q, are found in transmembrane helix 11. Surprisingly, the $\mathrm{H} 575 \mathrm{~L}$ variant reduced transport activity in a substrate specific manner. The reduction in rosuvastatin transport was in line with reduction in protein abundance, yet DCF transport was more drastically reduced, indicating the possibility of additional changes in substrate recognition or translocation. R580Q impaired the transport of both tested substrates significantly and is likely caused by decreased protein expression. Immunofluorescence, however, suggested no drastic change in neither abundance nor cellular localization of OATP1B1. Previously, alanine substitution at position 580 resulted in over $50 \%$ reduction in E3S transport (39). In a Western blot analysis of biotinylated HEK293 plasma membrane samples alanine reduced cell surface expression while lysine or histidine substitution did not change it (33). These results together with our novel findings suggest that a positively charged amino acid is necessary in the 580 position for proper function and localization of OATP1B1.

Overall, the studied variants in transmembrane helices 2, 4 and 11 appear to be crucial for proper membrane localization and function of OATP1B1, while the studied variants in helices 7 and 10 retained much of the function of the wild type OATP1B1.

The clinical significance of the SLCO1B1 c.521 T >C (V174A) genotype has prompted issuance of clinical prescribing guidelines (40). Individuals with two decreased-function alleles (low function phenotype) are considered to be at high myopathy risk during simvastatin treatment and the treatment is recommended to be initiated with a lower dosage. Based on our transport activity results, SNVs G76E, L193R, R580Q, could be categorized as low function variants, while R181C and $\mathrm{H} 575 \mathrm{~L}$ could be classified as intermediate function variants. T345M and $\mathrm{L} 543 \mathrm{~W}$ variants can be considered normal function variants, but P336R might lead to decreased plasma AUC values of rosuvastatin. Individuals with one decreased function allele or two intermediate function alleles could correspond to the intermediate function phenotype (40), which would also warrant caution when prescribing simvastatin. Due 
to the rarity of these variants (Table I), they are most likely to be found as heterozygotes. They could also be found as haplotypes with other SNVs such as V174A or N130D latter being very common in Sub-Saharan, East Asian and Oceanian populations (6). This could further alter their effects on transport activity. In vitro co-expression of P336R with N130D did not alter transport function compared to P336R expression only (26). When expressed with V174A, transport function was slightly improved compared to just V174A. Further research is required to establish the clinical effects of these phenotypes.

\section{CONCLUSIONS}

We have determined the transport activity and protein levels of nine variants of OATP1B1. To our knowledge, the G76E, R181C, T345M, H575L and R580Q variants were characterized for the first time. The in vitro transport activity of six of the studied variants $(\mathrm{G} 76 \mathrm{E}, \mathrm{V} 174 \mathrm{~A}$, R181C L193R, H575L and R580Q) was significantly reduced, two (T345M and L543W) had similar activity and one (P336R) variant had enhanced transport activity compared to the wild type OATP1B1. Overall, our results support previous studies suggesting that transmembrane helices 2, 4 and 11 are important for proper membrane localization and function of OATP1B1. While there was a trend between protein amount and activity, protein amount does not fully explain the observed transport activities, indicating involvement in substrate recognition or translocation for some of these residues. G76E, L193R and R580Q caused loss of function and thus could predispose the individuals with these genotypes to adverse effects of OATP1B1 substrate drugs similarly to V174A.

\section{FUNDING STATEMENT}

We are grateful for the funding provided by Svenska Kulturfonden, Medicinska Understödsföreningen for Liv och Hälsa, DRA Consulting oy, Instrumentarium Science Foundation and Finnish Cultural Foundation. The UEF Metabolomics laboratory is supported by Biocenter Finland and Biocenter Kuopio and the Drug Discovery and Chemical Biology Network and the Light Microscopy Unit are funded by Biocenter Finland. Dr. Melina M. Malinen received funding from the European Union's Horizon 2020 Research and Innovation program under the Marie Skłodowska-Curie grant agreement number 799510 .

\section{Acknowledgements}

We would like to thank Leena Pietilä, Olga Kaugonen, Noora Sjöstedt, Alli Tikkanen and Feng Deng for technical assistance. Dr. Yasuo Uchida is thanked for consultation on sample preparation of the proteomics samples. The staff at the Light Microscopy Unit in the Institute of Biotechnology within University of Helsinki is acknowledged for training in microscopy and image analysis. Dr. Vesa Niskanen is acknowledged for assistance in statistical analysis. We acknowledge the Drug Discovery and Chemical Biology Network for providing access to screening instrumentation.

Funding Open access funding provided by University of Helsinki including Helsinki University Central Hospital.

Open Access This article is licensed under a Creative Commons Attribution 4.0 International License, which permits use, sharing, adaptation, distribution and reproduction in any medium or format, as long as you give appropriate credit to the original author(s) and the source, provide a link to the Creative Commons licence, and indicate if changes were made. The images or other third party material in this article are included in the article's Creative Commons licence, unless indicated otherwise in a credit line to the material. If material is not included in the article's Creative Commons licence and your intended use is not permitted by statutory regulation or exceeds the permitted use, you will need to obtain permission directly from the copyright holder. To view a copy of this licence, visit http:/ / creativeco mmons.org/licenses/by/4.0/.

\section{REFERENCES}

1. Niemi M, Pasanen MK, Neuvonen PJ. Organic anion transporting polypeptide 1B1: a genetically polymorphic transporter of major importance for hepatic drug uptake. Pharmacol Rev. 2011;63(1):157-81.

2. European Medicines Agency. Guideline on the investigation of drug interactions. In: (CHMP) CfHMP, editor. 30 Churchill Place Canary Wharf London E14 5EU United Kingdom2012.

3. Administration FaD. Clinical Drug Interaction Studies Cytochrome P450 Enzyme- and Transporter-Mediated Drug InteractionsGuidance for Industry. In: (CDER) USDoHaHSCfDEaR, editor. 10001 New Hampshire Ave Silver Spring, MD, USA: Office of Communications, Division of Drug Information,; 2020.

4. Niemi M. Transporter pharmacogenetics and statin toxicity. Clin Pharmacol Ther. 2010;87(1):130-3.

5. Link E, Parish S, Armitage J, Bowman L, Heath S, Matsuda $\mathrm{F}$, et al. SLCO1B1 variants and statin-induced myopathy-a genomewide study. N Engl J Med. 2008;359(8):789-99.

6. Pasanen MK, Neuvonen PJ, Niemi M. Global analysis of genetic variation in SLCO1B1. Pharmacogenomics. 2008;9(1):19-33.

7. Ramsey LB, Bruun GH, Yang W, Trevino LR, Vattathil S, Scheet P, et al. Rare versus common variants in pharmacogenetics: SLCO1B1 variation and methotrexate disposition. Genome Res. 2012;22(1):1-8.

8. Prasad B, Evers R, Gupta A, Hop CE, Salphati L, Shukla S, et al. Interindividual variability in hepatic organic aniontransporting polypeptides and P-glycoprotein (ABCB1) protein expression: quantification by liquid chromatography tandem mass spectroscopy and influence of genotype, age, and sex. Drug metabolism and disposition: the biological fate of chemicals. 2014;42(1):78-88. 
9. Hagenbuch B, Stieger B. The SLCO (former SLC21) superfamily of transporters. Mol Aspects Med. 2013;34(2-3):396-412.

10. Adzhubei IA, Schmidt S, Peshkin L, Ramensky VE, Gerasimova A, Bork P, et al. A method and server for predicting damaging missense mutations. Nat Methods. 2010;7(4):248-9.

11. Ng PG, Henikoff S. SIFT: Predicting amino acid changes that affect protein function. Nucleic Acids Res. 2003;31(13):3812-4.

12. Omasits U, Ahrens CH, Müller S, Wollscheid B. Protter: interactive protein feature visualization and integration with experimental proteomic data. Bioinformatics (Oxford, England). 2014;30(6):884-6.

13. Karczewski KJ, Francioli LC, Tiao G, Cummings BB, Alföldi J, Wang Q, et al. The mutational constraint spectrum quantified from variation in 141,456 humans. Nature. 2020;581 (7809):434-43.

14. El-Sheikh AAK, van den Heuvel JJMW, Koenderink JB, Russel FGM. Interaction of Nonsteroidal Anti-Inflammatory Drugs with Multidrug Resistance Protein (MRP) 2/ABCC2- and MRP4/ABCC4-Mediated Methotrexate Transport. J Pharmacol Exp Ther. 2007;320(1):229-35.

15. Tikkanen A, Pierrot E, Deng F, Sánchez VB, Hagström M, Koenderink JB, et al. Food Additives as Inhibitors of Intestinal Drug Transporter OATP2B1. Mol Pharm. 2020;17(10):3748-58.

16. Sjostedt N, van den Heuvel J, Koenderink JB, Kidron H. Transmembrane Domain Single-Nucleotide Polymorphisms Impair Expression and Transport Activity of ABC Transporter ABCG2. Pharm Res. 2017;34(8):1626-36.

17. Uchida Y, Tachikawa M, Obuchi W, Hoshi Y, Tomioka Y, Ohtsuki S, et al. A study protocol for quantitative targeted absolute proteomics (QTAP) by LC-MS/MS: application for inter-strain differences in protein expression levels of transporters, receptors, claudin- 5 , and marker proteins at the blood-brain barrier in $\mathrm{ddY}, \mathrm{FVB}$, and C57BL/6J mice. Fluids and Barriers of the CNS. 2013;10(1):21.

18. Gynther M, Proietti Silvestri I, Hansen JC, Hansen KB, Malm T, Ishchenko Y, et al. Augmentation of Anticancer Drug Efficacy in Murine Hepatocellular Carcinoma Cells by a Peripherally Acting Competitive N-Methyl-d-aspartate (NMDA) Receptor Antagonist. J Med Chem. 2017;60(23):9885-904.

19. Huttunen J, Gynther M, Vellonen KS, Huttunen KM. L-Type amino acid transporter 1 (LAT1)-utilizing prodrugs are carrier-selective despite having low affinity for organic anion transporting polypeptides (OATPs). Int J Pharm. 2019;571:118714.

20. de Graan AJ, Lancaster CS, Obaidat A, Hagenbuch B, Elens L, Friberg LE, et al. Influence of polymorphic OATP1B-type carriers on the disposition of docetaxel. Clinical cancer research: an official journal of the American Association for Cancer Research. 2012;18(16):4433-40.

21. Li N, Hong W, Huang H, Lu H, Lin G, Hong M. Identification of amino acids essential for estrone-3-sulfate transport within transmembrane domain 2 of organic anion transporting polypeptide 1B1. PLoS One. 2012;7(5):e36647.

22. Pasanen MK, Fredrikson H, Neuvonen PJ, Niemi M. Different effects of SLCO1B1 polymorphism on the pharmacokinetics of atorvastatin and rosuvastatin. Clin Pharmacol Ther. 2007;82(6):726-33.

23. Pasanen MK, Neuvonen M, Neuvonen PJ, Niemi M. SLCO1B1 polymorphism markedly affects the pharmacokinetics of simvastatin acid. Pharmacogenet Genomics. 2006;16(12):873-9.
24. Nies AT, Niemi M, Burk O, Winter S, Zanger UM, Stieger $\mathrm{B}$, et al. Genetics is a major determinant of expression of the human hepatic uptake transporter OATP1B1, but not of OATP1B3 and OATP2B1. Genome medicine. 2013;5(1):1.

25. Tirona RG, Leake BF, Merino G, Kim RB. Polymorphisms in OATP-C: identification of multiple allelic variants associated with altered transport activity among European- and AfricanAmericans. J Biol Chem. 2001;276(38):35669-75.

26. Kameyama Y, Yamashita K, Kobayashi K, Hosokawa M, Chiba K. Functional characterization of SLCO1B1 (OATP-C) variants, SLCO1B1*5, SLCO1B1*15 and SLCO1B1*15+C1007G, by using transient expression systems of HeLa and HEK293 cells. Pharmacogenet Genomics. 2005;15(7):513-22.

27. Iwai M, Suzuki H, Ieiri I, Otsubo K, Sugiyama Y. Functional analysis of single nucleotide polymorphisms of hepatic organic anion transporter OATP1B1 (OATP-C). Pharmacogenetics. 2004;14(11):749-57.

28. Nozawa T, Nakajima M, Tamai I, Noda K, Nezu J, Sai Y, et al. Genetic polymorphisms of human organic anion transporters OATP-C (SLC21A6) and OATP-B (SLC21A9): allele frequencies in the Japanese population and functional analysis. J Pharmacol Exp Ther. 2002;302(2):804-13.

29. Crowe A, Zheng W, Miller J, Pahwa S, Alam K, Fung KM, et al. Characterization of Plasma Membrane Localization and Phosphorylation Status of Organic Anion Transporting Polypeptide (OATP) 1B1 c.521 T>C Nonsynonymous Single-Nucleotide Polymorphism. Pharmaceutical research. 2019;36(7):101.

30. Burt HJ, Riedmaier AE, Harwood MD, Crewe HK, Gill KL, Neuhoff S. Abundance of Hepatic Transporters in Caucasians: A Meta-Analysis. Drug metabolism and disposition: the biological fate of chemicals. 2016;44(10):1550-61.

31. Ho RH, Tirona RG, Leake BF, Glaeser H, Lee W, Lemke CJ, et al. Drug and bile acid transporters in rosuvastatin hepatic uptake: function, expression, and pharmacogenetics. Gastroenterology. 2006;130(6):1793-806.

32. Meier-Abt F, Mokrab Y, Mizuguchi K. Organic anion transporting polypeptides of the OATP/SLCO superfamily: identification of new members in nonmammalian species, comparative modeling and a potential transport mode. J Membr Biol. 2005;208(3):213-27.

33. Weaver YM, Hagenbuch B. Several conserved positively charged amino acids in OATP1B1 are involved in binding or translocation of different substrates. J Membr Biol. 2010;236(3):279-90.

34. Michalski C, Cui Y, Nies AT, Nuessler AK, Neuhaus P, Zanger UM, et al. A naturally occurring mutation in the SLC21A6 gene causing impaired membrane localization of the hepatocyte uptake transporter. J Biol Chem. 2002;277(45):43058-63.

35. Wang X, Liang Y, Fang Z, Huang J, Hong M. The intracellular NPxY motif is critical in maintaining the function and expression of human organic anion transporting polypeptide 1B1. Biochim Biophys Acta. 2019;1861(6):1189-96.

36. Nishizato Y, Ieiri I, Suzuki H, Kimura M, Kawabata K, Hirota T, et al. Polymorphisms of OATP-C (SLC21A6) and OAT3 (SLC22A8) genes: consequences for pravastatin pharmacokinetics. Clin Pharmacol Ther. 2003;73(6):554-65.

37. Morimoto K, Oishi T, Ueda S, Ueda M, Hosokawa M, Chiba $\mathrm{K}$. A novel variant allele of OATP-C (SLCO1B1) found in a Japanese patient with pravastatin-induced myopathy. Drug Metab Pharmacokinet. 2004;19(6):453-5.

38. Furihata T, Satoh N, Ohishi T, Ugajin M, Kameyama Y, Morimoto K, et al. Functional analysis of a mutation in the SLCO1B1 gene (c.1628T $>$ G) identified in a Japanese patient 
with pravastatin-induced myopathy. The pharmacogenomics journal. 2009;9(3):185-93.

39. Hong W, Wu Z, Fang Z, Huang J, Huang H, Hong M. Amino Acid Residues in the Putative Transmembrane Domain 11 of Human Organic Anion Transporting Polypeptide 1B1 Dictate Transporter Substrate Binding, Stability, and Trafficking. Mol Pharm. 2015;12(12):4270-6.

40. Ramsey LB, Johnson SG, Caudle KE, Haidar CE, Voora D, Wilke RA, et al. The clinical pharmacogenetics implementation consortium guideline for SLCO1B1 and simvastatin-induced myopathy: 2014 update. Clin Pharmacol Ther. 2014;96(4):423-8.

Publisher's Note Springer Nature remains neutral with regard to jurisdictional claims in published maps and institutional affiliations. 